\title{
LOS CUIDADOS FAMILIARES \\ DE SALUD EN LAS PERSONAS \\ ANCIANAS Y LAS POLITICAS SOCIALES
}

\author{
María Teresa Bazo \\ Universidad del País Vasco
}

\section{Carmen Domínguez-Alcón}

Universidad de Barcelona

Las personas ancianas en España, al igual que en otras sociedades industrializadas, son en su mayoría independientes y no requieren ayuda para la realización de las actividades de la vida cotidiana (Alonso, 1990: 229; Gobierno Vasco, 1995: 45; Vallés y Cea, 1994: 869; Bazo, 1990: 35; Encuesta de Salud de Barcelona 1992-93: 13). Se alcanza cada vez edades más avanzadas en mejor estado de salud, pero el hecho de que se viva más conlleva mayores probabilidades de padecer enfermedades crónicas e invalidantes, y algunas personas sí necesitan de la ayuda de otras. Aunque la edad no es la variable decisiva en cuanto a la pérdida de la salud e independencia, sin embargo, existe una asociación entre ambas variables.

La mayor parte de las personas enfermas y discapacitadas recibe el cuidado requerido fuera del sistema sanitario, de forma no institucional, proporcionado por sus familias, y diferentes estudios confirman que, en general, son las mujeres quienes realizan esas tareas. En 1984 se estimaba que, en España, una de cada diez amas de casa tenía una persona discapacitada entre sus parientes próximos. En 1990, la Encuesta de Nuevas Demandas confirma la estimación que sólo un 10 por 100 de discapacitados recibe institucionalmente el cuidado requerido. Cada mil amas de casa cuidaban 76 personas discapacitadas, de los que dos terceras partes llevaban más de seis años en esa situación (Durán, 1992: 67 y ss.). No obstante, casi un tercio de personas de más de 65 años se cuidan a ellas mismas, el 28,7 por 100 de varones y un 27,5 por 100 de muje-

\section{Reis}


res, según indican los datos de la Encuesta de Salud de Barcelona 1992-93 (p. XXII), siendo en segundo lugar una mujer de la casa quien los cuida.

La investigación en la que se basa el presente artículo, sobre solidaridad familiar y vejez, ha sido realizada en el País Vasco, Madrid y Cataluña (Bazo y Domínguez-Alcón, 1995); centra la atención específicamente en los cuidados de salud en la familia para las personas ancianas, y los resultados obtenidos confirman ampliamente la hipótesis de que la familia es la principal proveedora de cuidados y que en el grupo familiar las mujeres asumen mayormente esa responsabilidad, aunque la presencia de varones realizando tareas de cuidados va siendo progresivamente más frecuente.

En la investigación se realiza una aproximación a diferentes grupos de personas, en su mayoría cuidadoras principales de personas ancianas con graves problemas de dependencia. Se utiliza metodología cualitativa. La entrevista en profundidad semiestructurada se aplica a una muestra amplia que incluye algo más de cien personas en las tres comunidades autónomas: Madrid, País Vasco y Cataluña. También se realizan tres entrevistas de grupo (una en cada comunidad) a personas pertenecientes a la Asociación de Familiares de Enfermos de Alzheimer.

En la actualidad, el análisis de la ancianidad tiene en cuenta, más que los aspectos patológicos, la salud y calidad de vida positiva en la vejez (Bazo, 1992a). Ser anciano/a no significa ser enfermo; sin embargo, muchas personas ancianas necesitan, temporal o definitivamente, ser atendidas sanitaria y socialmente. La mayor parte de las personas mayores no se sienten enfermas, ni viejas, ni solas, ni deprimidas; incluso algunas de ellas son agentes activos, y realizan valiosas contribuciones a la sociedad (SECOT, 1995; Moen et al., 1992; Bazo, 1994). Pero es igualmente cierto que algunas personas ancianas sufren por algunas o todas esas causas, y su calidad de vida se ve notablemente mermada en su vejez.

Una parte considerable de los subgrupos de la población de más edad son los que sufren una mayor incidencia y prevalencia de enfermedades crónicas y discapacitantes, que afectan en mayor proporción a las mujeres, de las que casi la mitad son viudas y que, por no haber cotizado en su mayoría durante la edad activa, hoy subsisten con pensiones de viudedad o asistenciales, lo cual influye negativamente para hacer frente a las situaciones de dependencia y enfermedad.

La vejez es una construcción cultural. La actual es consecuencia de políticas y estilos de vida anteriores. La próxima vejez la estamos construyendo ahora. Por eso es relevante tener en cuenta las diferentes dimensiones e implicaciones de cada una de las situaciones que se analizan en la investigación que se comenta. Aumenta la invalidez, discapacidad y minusvalía, pero el análisis de costos sobre la atención a todas y cada una de esas situaciones no puede realizarse únicamente desde una perspectiva de mercado. En cuanto al gasto sanitario, aunque las personas mayores consumen más servicios y medicamentos que otros grupos de edad, las pautas de consumo no son homogéneas para 
todo el grupo de personas mayores. Se concentra en las edades más avanzadas y en el período previo a la muerte. Es un aspecto que se debate actualmente (Scitovsky, 1994: 586), dado que la mayoría de los análisis ponen el énfasis en el coste de la alta tecnología, olvidando o no considerando el número de personas que mueren en su casa o en otras instituciones y que no están sometidas a tratamientos costosos.

Las condiciones generales de vida y trabajo están cambiando aceleradamente y no resulta descabellado pensar en prolongar la edad de jubilación, ya que el deterioro severo de la salud llega más tarde. El volumen de cuidados de salud que se requería anteriormente se experimentará por las personas a una edad más tardía, pero las personas en un momento dado necesitarán los cuidados. De ahí la importancia, para la política de salud, de la investigación e información en los cambios en los niveles de salud en la estructura de edades cambiante, e investigar sobre el comienzo y duración de las enfermedades crónicas.

En el futuro se prevé que el índice de dependencia aumente y que cambie su estructura, recayendo el mayor peso sobre las personas de más edad, al tiempo que disminuye el de las más jóvenes. Pero, si se prolonga la edad de jubilación, la definición del concepto y los resultados de esa relación variarán y puede que en las próximas décadas el cambio no resulte tan significativo proporcionalmente. Sin embargo, no se alterará el hecho del incremento potencial del período de dependencia en las personas ancianas previo a la muerte.

Un gran tema de debate en la elaboración de las políticas y provisión de los servicios sociales es el cuidado de las personas ancianas y, subsecuentemente, el reparto de las responsabilidades. Las reflexiones y el cuestionamiento que se plantean hoy son los del paso del Estado del Bienestar a lo que se denomina «sociedad del bienestar», y sus consecuencias desde una perspectiva de mayor alcance que la económica y de gestión, como recientemente se expresa (Castiñeira, 1995: 4). En sociedades con un Estado del Bienestar más desarrollado que en la nuestra se entiende que los servicios públicos vienen a ser un aumento reciente a la ayuda que pueden recibir las personas, que se sobrepone a aquélla, y que transforma los viejos modelos de cuidado informal provisto por la familia y los vecinos.

En la actual coyuntura, donde se cuestiona la continuidad, al menos tal como ha llegado a desarrollarse, del Estado del Bienestar, se entiende que la cuestión de la provisión de los cuidados a las personas más ancianas —que son las que proporcionalmente más aumentan- es una cuestión de vital importancia. Se hace un llamamiento al sector informal, pero se teme que los cambios familiares operados neutralicen el potencial de cuidados que puede proporcionar en el próximo futuro.

Se considera que existe una interdependencia entre los sectores informal y formal, ya que el sector formal sería incapaz de asumir los cuidados de todas las personas dependientes, y porque el sector informal es en parte función de la oferta y distribución de los servicios sociales y de las políticas económicas y sociales del Estado (Qureshi y Walker, 1991). Sin embargo, en España, la esca- 
sez de los servicios de cuidado comunitario provistos por el sector público y la amplitud de los servicios informales provistos por la familia cuestionan tal interdependencia.

El sector formal aparece en la mayor parte de las comunidades autónomas como un mero apéndice debido a su débil impacto social y la escasa demanda, en relación a las necesidades, realizada por parte de las familias. Una causa que influye de manera considerable en esa situación es el sentimiento generalizado entre la población de la asunción del cuidado de las personas mayores de la familia como parte de los deberes familiares. Actitudes que incluso parte de la generación de jóvenes ha interiorizado (Bazo, 1994).

El cuidado familiar se ha basado en el tiempo y el trabajo de las mujeres, que se supone es libre en términos económicos. Actualmente se entiende que la disminución del tamaño familiar, la incorporación de las mujeres al mundo laboral, sus niveles más altos de instrucción, el incremento en el número de divorcios, de familias monoparentales, la cohabitación, la formación de familias reconstituidas, y los cambios que todo ello conlleva en los modelos tradicionales de familia en las sociedades denominadas occidentales, ponen en peligro la continuidad de dichos cuidados.

Las políticas y los servicios sociales se elaboran teniendo en cuenta una concepción determinada de la familia y la comunidad. La familia se ha entendido compartiendo responsabilidades entre sus miembros y ofreciéndose mutuamente contraprestaciones, como algo propio de las «obligaciones familiares» (Domínguez-Alcón, 1994). El modo de asumir las obligaciones familiares, de compartir las situaciones de crisis y dificultad, la forma de manifestar la solidaridad y la ayuda mutua, incluyen diferentes y complejas dimensiones (Bonvalet et al., 1994; Roussel, 1994). La concepción de la familia sobre la que se conciben las políticas sociales se basa en una estructura en la que el varón es el proveedor de los ingresos y la mujer la proveedora de los cuidados y el servicio. La diferenciación de roles en la familia lleva a las mujeres a especializarse como amas de casa y madres. Se considera que su tiempo empleado en el desempeño de tales roles es libre en términos de disponibilidad y costo (Allan, 1990: 17). Sin embargo, las transformaciones a nivel de la familia tienen diferentes implicaciones según el género, las relaciones entre los diferentes miembros de la familia y de parientes más próximos, y es por ello que resulta necesario profundizar en el tema.

En cuanto a la investigación realizada por las autoras en Madrid, el País Vasco y Cataluña, puede decirse que la mayoría de las personas cuidadoras son mujeres, al igual que ocurre en el universo a que corresponde la muestra. Son esposas e hijas. En las muestras de Madrid y el País Vasco la proporción es similar; en cambio, en Cataluña la presencia de hijas es algo mayor entre las personas cuidadoras. La participación de nueras es más reducida, así como la atención a otros familiares distintos del cónyuge o padres; en algún caso se cuida a una tía o prima y, excepcionalmente, a una persona que no es de la familia, aunque con vínculos de amistad fuertes. 
La presencia de varones cuidadores existe, pero es testimonial. Son esposos que cuidan a su pareja, o hijos, generalmente solteros, que cuidan al padre o madre. En todos los casos, los cuidadores varones analizados son a su vez mayores, de entre 60 y 70 años o algo más. De todos modos, al tratarse de muestras no aleatorias y de una investigación cualitativa, estos datos no sirven, obviamente, para realizar inferencias.

Las edades de las mujeres cuidadoras son variadas, desde los 20 años hasta casi los 80. Sin embargo, hay algunas diferencias. En Cataluña se concentran en una franja de edad entre los 55 y los 76 años, salvo algún caso de mujeres algo más jóvenes. En cambio, en la muestra de Madrid se diferencian claramente entre las cuidadoras dos grupos: uno más joven, cuyas edades oscilan entre los 37 y alrededor de los 50 años, y otro grupo de cuidadoras ancianas cuyas edades están comprendidas entre los 70 y los 80 años.

Las diferencias de edad que se observan están en relación con el parentesco que mantienen las personas cuidadoras respecto a quienes cuidan. En Madrid, el grupo de esposas es más numeroso entre las cuidadoras, mientras que en Cataluña y en el País Vasco son las hijas el grupo más nutrido. Se observa también la presencia de algún esposo, varias esposas e incluso, en el País Vasco, algunas nietas y nietos. Las edades de las personas que reciben los cuidados están comprendidas entre los 70 y los 93 años; en algún caso la edad es inferior. De todas formas, la mayoría de las personas que reciben cuidados se acerca a la franja de edad más alta, de 86 años en adelante.

Las situaciones de las personas que reciben cuidados parecen más graves y de una dependencia más severa en la muestra de Madrid. En la mayoría de casos se trata de personas inválidas, afectadas de incontinencia, y es frecuente la presencia de otros trastornos discapacitantes, ceguera, ex-alcoholismo u otros. En la muestra de Cataluña, las situaciones que las personas cuidadoras manifiestan requieren a menudo la presencia permanente y cerca de la persona cuidadora, pero en la explicitación de las situaciones vividas no se expresan de forma tan intensa como en Madrid la afectación biológica y las fuertes demandas al cuidador/a para la realización de las actividades de la vida cotidiana, aunque sí hay algún caso de dependencia muy acusada.

Un aspecto que la investigación ha puesto de manifiesto es la solidaridad familiar: "Estoy preocupada porque ella necesita compañia, necesita a la familia; puede que tengamos amigos, pero la familia hace mucho." "Al enfermo se le quiere. Cuando es una persona que quieres no te lo sacas de encima; yo lo cuidaría hasta que no pudiera más, hasta el límite mio." "Yo estoy dispuesta por mi madre y por mi suegro a dejarlo todo, a cuidarlos a ellos."

Con expresiones como las anteriores o con matices similares, en la gran mayoría de los casos se expresa la solidaridad familiar, que se manifiesta con fuerza y rotundidad en el deseo y en la práctica habitual de conservar a las personas ancianas, dependientes y enfermas en el hogar. Se rechaza de forma unánime la institucionalización, salvo en los casos en que se produce un trastorno circunstancial o en los casos extremos en que asumir el cuidado en el hogar es 
prácticamente imposible. Existe la solidaridad y se expresa de esa forma una sensibilidad hacia aceptar el deber de ayuda, querer hacerlo y llevarlo a la práctica.

Se expresa el deseo de cuidar a los familiares en el hogar: "Yo sé que en toda la vida no han querido ir a una residencia y siempre han dicho que en su casita; ahora sacarlos sería matarlos, y no lo quiero." "Si la llevara a alguna parte estaría yo peor que ella, pudiendo cuidarla yo." "Mientras yo pueda, nadie de mi familia irá a un sitio a que le cuide otra persona." "Se tienen en el domicilio aunque cuesten sacrificios. Yo no creo que te tengas que desprender de un enfermo, ni de un anciano; al contrario. Cuando uno no está bien es cuando necesita el cariño de las personas que le rodean."

De esas formas o parecidas se manifiesta por parte de los familiares el deseo de que las personas ancianas, dependientes y enfermas deben permanecer en el hogar. El sentimiento es intenso y general, hasta el punto de que en alguna ocasión fuerza incluso a que la familia desinstitucionalice a la persona dependiente. Los testimonios recogidos en Madrid, País Vasco y Cataluña reflejan ampliamente ese aspecto. Las expresiones son variadas y ofrecen un rico conjunto de matices con el que los familiares expresan, con el mayor convencimiento, ese sentimiento natural de atender y cuidar en el hogar a la persona anciana enferma y dependiente.

Existe, asimismo, una idea de correspondencia, de deber con los padres ancianos: "Mis padres han dado la vida por mí." "Sobre todo si ellos se han portado bien contigo, no porque lo tengas que hacer por compensación, sino que va de cajón, ya es así." "Yo creo que es una obligación." "No me doy cuenta de que estoy haciendo nada extraordinario, lo veo normal, aunque es muy duro." "Yo prefiero hacer todo lo que estoy haciendo ahora." "Les quiero con locura. Estoy pendiente y quiero hacerlo.»

De esa manera o similar se manifiestan las personas cuidadoras en los tres territorios en los que se realiza la investigación. La percepción de la situación es variada, pero la orientación es común. Cuidar a un familiar enfermo, anciano y dependiente significa cumplir con un deber, existe la obligación de hacerlo, aunque al mismo tiempo se cuida con cariño y afecto. Está presente la manifestación de reciprocidad, la compensación y el sentimiento de no defraudar las expectativas de la persona que recibe los cuidados. La reciprocidad es una noción que se identifica entre los componentes de los cuidados (Mayerof, 1972: 36) y que se manifiesta fundamental en la expresión de las personas cuidadoras.

Se observa que las personas cuidadoras sienten la necesidad de cumplir con las obligaciones familiares, y las respuestas que ofrecen son distintas. Existe acuerdo en que hay que hacer algo, pero no hay acuerdo en qué exactamente. Ese es un aspecto que señalan algunos estudios (Finch y Masson, 1993: 96106). Las respuestas frente al sentimiento de obligación son diversas, desde un total compromiso de la persona cuidadora a un cierto desentendimiento en otros familiares. Puede tratarse de una ayuda material intensa, dedicando gran 
cantidad de tiempo, ayudando a la persona dependiente a hacer frente a las actividades de la vida cotidiana. Hay ocasiones en las que por parte de otros familiares se ofrece una ayuda material, otras actividades, pero a una cierta distancia, aunque manifestando apoyo y soporte que se ha visto es tan necesario para la persona cuidadora en todos los casos. Incluso se acepta peor la falta de soporte familiar por parte de la persona cuidadora que la propia situación de cuidados, aunque ésta sea difícil.

El compromiso parece ser más fuerte por parte de las mujeres, especialmente de las hijas, y ésa es una característica común en Madrid, País Vasco y Cataluña. La calidad anterior de las relaciones entre la persona cuidadora y la que recibe los cuidados no parece influir a la hora de hacer frente a las situaciones de cuidados. Como se ha observado en otras investigaciones (Ungerson, 1987: 57; Walker, 1991: 106), entendemos que lo sociológicamente interesante es que en nuestra investigación se observa una construcción social del rol de cuidadora, basada en creencias tradicionales acerca de las funciones protectoras de la familia, y sobre todo de las mujeres, como las proveedoras naturales de cuidados. Entendemos que ésa es la razón latente, no expresada, de por qué cuidan las mujeres, ya que lo hacen sientan o no amor por la persona cuidada, existan o no sentimientos de reciprocidad, hayan sido las relaciones previas buenas o malas, existan posibilidades materiales adecuadas o no (disponibilidad de tiempo, condiciones de la vivienda). Las personas cuidan por una suerte de imperativo social (aunque, además, se sienta amor por la persona cuidada). Pero esa coerción moral (durkheimiana) no suele percibirse, precisamente por como ha sido interiorizada en los procesos de socialización. Y todavía la familia sigue transmitiendo esos valores a las generaciones jóvenes (Bazo, 1994). Es por lo que puede predecirse que en el futuro las personas pueden sufrir serios conflictos.

Se ha puesto especial énfasis en la perspectiva de la persona cuidadora y en los lazos familiares, dando menos importancia a otros aspectos sobre los que convendría igualmente profundizar, como, por ejemplo, las posibilidades de elección para la persona que recibe los cuidados en cuanto al entorno en que prefiere estar, o las personas con quienes desea convivir o tener cerca. En ocasiones es preciso hacer frente a aspectos estructurales de cambios de domicilio para estar cerca de los hijos e hijas. Es igualmente patente en varios casos la sensibilidad de las hijas que desean respetar el entorno habitual de sus padres, manteniéndolos en el hogar de la persona enferma, aunque eso represente para la persona cuidadora una desorganización de sus roles, en su propio hogar, hábitos y/o familia.

Son diversos los sentimientos expresados: "Me ha compensado cuidarle.» "Me ha compensado porque me ha dado tranquilidad." "Expresa mucho agradecimiento, me quiere muchisimo, me lo expresa dándome besos." "Cualquier cosa que le hacias te la agradecía." "Hay personas mayores que son dulces, pero ella no, no puede ser dulce; es muy áspera de carácter, y eso se acusa." "Te exigen y no son agradecidos." "He tenido que aguantarme yo para calmarla a ella." 
La expresión de los sentimientos en la relación de las personas cuidadoras con las receptoras de cuidados es abundante. Se manifiestan en un rico conjunto de matices diferentes experiencias, predominantemente gratificantes, relacionadas con el cuidar. De todas formas, las relaciones son con frecuencia complejas, y se expresan incluso sentimientos contradictorios. Se tiende a justificar comportamientos poco amables de quien recibe los cuidados. Se manifiesta afecto, pero también impotencia y resignación. Asimismo, en el ánimo de los familiares aparecen a menudo sentimientos de desesperación, de soledad, ante la ruptura de comunicación con la persona enferma, y, aunque de forma minoritaria, también se manifiesta una mala convivencia.

Son muchas las tareas que las personas cuidadoras deben realizar: "Tengo que estar siempre pendiente de él, se lava solo y come solo, pero está casi ciego y es incontinente." "Ahora no se entera de casi nada, hay que hacerle todo, pero ha estado muy agresiva; nuestro padre, que ha muerto hace cinco meses, estaba todavia peor que ella." "Tenias que hacerle todo lo que necesita una persona, y por la noche igual, te llamaba seis o siete veces por la noche." "Yo he pasado muchisimo, le hacía todo, le limpiaba, le afeitaba, y yo con mis muletas a trancas y barrancas; hemos pasado lo que nadie, no quiero ni recordarlo."

La expresión de los testimonios ilustra ampliamente que cuidar a una persona anciana y dependiente supone a menudo la realización de diferentes actividades que pueden implicar tipos distintos de carga física o mental para la persona cuidadora y para los miembros que constituyen la red familiar. La situación de autonomía relativa existe en varios casos y la persona anciana necesita únicamente de la presencia física de alguien que esté cerca, disponible, más que la realización de cuidados concretos. En los casos de dependencia leve, la persona anciana se las arregla sola, salvo en alguna circunstancia específica. Pero existen otras manifestaciones de dependencia severa, y muy severa, que recogen ampliamente los testimonios de las personas cuidadoras. Situaciones en las que la persona cuidadora tiene una intensa y continuada participación en la ayuda para las actividades de la vida cotidiana, y la persona anciana está completamente impedida o con fuerte afectación y limitación en su movilidad y autonomía. En algunos casos, en la muestra estudiada coinciden dos personas con dependencia severa en el mismo hogar y al mismo tiempo, y ello se prolonga durante un período de duración considerable. La dedicación intensa y atención permanente tiene una fuerte presencia en la muestra de Madrid.

En varios casos, la actividad cuidadora se realiza al mismo tiempo que la actividad económica: "Trabajaba porque teníamos un comercio, pero cerramos. ¿Cómo iba a trabajar en esas circunstancias?" "Yo trabajo por la mañana y tengo una persona que cuida a mi madre, pero hay que pagarla." "Trabajaba cuando cuidaba a mi padre, sacando fuerzas de flaqueza." "Me desanimo mucho, porque no llego a todo."

Las manifestaciones sobre la repercusión de la situación de cuidados en relación con el trabajo de la mujer son evidentes en varias entrevistas. La 
imposibilidad de trabajar, tener que dejar el trabajo por dificultades de encontrar ayuda, no poder suplir a la cuidadora, son frecuentes. Se indican diferentes vertientes y aspectos relacionados con el trabajo de la mujer, desde la realización del mismo como ayuda económica familiar a la expresión de un sentido del trabajo más cercano al desarrollo y equilibrio personal para la cuidadora. Todos ellos son aspectos igualmente presentes en el País Vasco y Cataluña.

Resulta pertinente resaltar un aspecto, y es el relativo a la, en muchos casos, exclusión social de la persona cuidadora, a su aislamiento social en muchas ocasiones a causa de su actividad cuidadora: «El cuidar a mis padres supuso un cambio total en mi vida." "La vida nuestra es muy dura, no salgo, ni veraneo, ni nada. No he tenido vida ni alegría." "No teníamos libertad.» "El cuidado era diario, mañana y tarde, sábados y domingos." "Si no tienes una persona que te venga a ayudar siempre estás subordinada, sin poderte mover." "Tengo que estar siempre pendiente de él." "No le puedo dejar solo." "Lo he pasado muy mal, me tenía en un puño; regresaba de trabajar y cuando venia iba pensando en lo que me iba a encontrar."

Las expresiones relacionadas con la restricción de libertad, individual y familiar, son una característica general y común en todos los casos. Al igual que la supeditación intensa de la persona cuidadora, alteración de su vida personal y grave impacto sobre la salud de la persona cuidadora. Hay acuerdo unánime en que la vida cambia y que se altera completamente la vida familiar. Se constata un empobrecimiento de las relaciones de las personas cuidadoras con el entorno. A ello se añade el que las personas cuidadoras están insertas en una red familiar y de relaciones muy reducida, de pocos parientes disponibles. La situación de cuidados a la que deben hacer frente limita más, si cabe, las posibilidades de relación, ya que frecuentemente se alarga durante años y es progresivamente más compleja y absorbente para la persona cuidadora. Esa situación la va aislando de todo y de todos a medida que transcurre el tiempo.

El cuidado en la comunidad se plantea de esa forma asociado a la solidaridad familiar, con una especial significación respecto del espacio privado de la mujer, ligado a formas de provisión de cuidados cuyos esquemas no están todavía lo suficientemente organizados como recursos de la comunidad, y que resultan progresivamente excluyentes para la persona cuidadora.

La salud física y psíquica de la persona cuidadora resulta a menudo dañada por la prolongada situación del cuidado: "Personalmente me ha repercutido muchisimo en mi vida." "La enfermedad de mi marido me ha agriado la vida." "Para atenderle he estado seis años contra reloj." "Cuando estuvieron los dos enfermos en casa si estuve mal, me encontraba mal, agotada, nerviosa, con todo no se puede." "Mi estado de ánimo es muy malo, no puedo con todo." "Estaba hundida, con muchas ganas de llorar." "Era mucho, estaba agotada, llegaba a casa y les chillaba a los niños.» "Duermo muy mal.» "Encuentro que todo es un agobio, y además este dolor de cabeza." "Estoy reventado, ya no puedo más."

Es constante la mención por parte de las personas cuidadoras al cansancio físico, agotamiento, desánimo, así como los trastornos del descanso y sueño, 
que son comunes en la mayoría de los casos. En algunos incluso la afectación de la salud de la persona cuidadora es más severa, y se relatan episodios de depresión o de dificultad muy intensa para asumir la situación.

El impacto real sobre la salud de las personas cuidadoras, la percepción unánime de que la vida les cambia por el hecho de hacer frente a la situación de cuidados, junto con los sentimientos de impotencia y resignación, son características comunes en casi todas las situaciones analizadas en la investigación. A partir de esos factores citados presentes de forma casi constante en las situaciones de cuidados, las estrategias familiares para hacerles frente son diversas, y la información pormenorizada que aportan las entrevistas realizadas son bien ilustrativas al respecto.

Se puede decir que en la investigación se detecta que las personas cuidadoras no disponen de ayudas suficientes, y ni siquiera de una información básica sobre las posibilidades de obtenerlas, e incluso sobre el desarrollo de la enfermedad de sus familiares: "Las personas que se dedican a cuidar enfermos no tienen suficiente ayuda." "Existen pocas ayudas institucionales." "Si no pagas no encuentras a nadie que te ayude." "La ayuda institucional es dificilísima, incluso la particular ponen pegas." "La situación es mala para los que tienen lo de mi mujer, yo estoy solo, con sesenta y seis años, y tengo que levantarla y hacerle la cama, y todas las cosas que hay que hacer." "No me podian enviar a nadie en todo el mes, ¿para qué voy a ir otra vez? ¿para que me digan lo mismo?"

Tanto en la muestra de Madrid como en las del País Vasco y Cataluña es común e intenso el sentimiento que expresan las familias de que no existen suficientes ayudas. Se constata, además, un desconocimiento bastante generalizado sobre las posibilidades de acceso a las escasas ayudas disponibles, y la convicción e incluso la experiencia de la dificultad o imposibilidad de obtención de las mismas. A pesar de ello, no se expresan muchas quejas. Se menciona, eso sí, con frecuencia la escasez de ayuda moral, material e incluso mecánica (sillas de ruedas u otras ayudas mecánicas), la falta de atención, coordinación y, en algún caso, desatención por parte de los sistemas sanitario y social.

Los criterios sobre los que se conceden las ayudas son igualmente motivo de comentario. En general, por su poca flexibilidad y su casi exclusiva consideración de los aspectos económicos. En varios casos existe una gran precariedad económica, mientras que en otros la necesidad económica no es la más perentoria y sí lo son otras como la ayuda material, mecánica, personal o de apoyo y soporte moral, que están al descubierto y sin posibilidad de que las condiciones de contexto, estructurales o familiares posibilite hacerles frente. Se observa que la aplicación de criterios exclusivamente económicos de elegibilidad discriminan cada vez más a ciertos grupos sociales pertenecientes a la clase media baja, que superan los límites económicos establecidos administrativamente, pero no pueden comprar los servicios privados.

Atender por parte de la familia a una persona anciana enferma y discapacitada se vive con frecuencia como un problema, muy especialmente por la exigencia progresiva que implican las situaciones que se analizan en la presente 
investigación. Todo ello hace pensar que, probablemente, ni en Madrid, País Vasco o Cataluña la situación será igual en el futuro. La atención no podrá seguir recayendo casi en exclusiva sobre la familia, ni sobre la mujer. En varios de los casos analizados los miembros de la familia nuclear comparten el cuidado, pero se ha constatado que la red de familiares disponibles en la familia extensa es reducida.

Los detalles que la investigación aporta respecto a las dificultades que conlleva compartir el cuidado, a pesar de poder contar con algunos miembros de la familia, junto con la presencia de desatención, desentendimiento y falta de apoyo de la familia en otros casos, muestra claramente la fragilidad del sistema. De todas formas, se ha podido constatar la fuerte convicción y el deseo de mantener en el hogar a la persona que necesita atención, pero las condiciones en las que se desarrolla la atención y la provisión de los cuidados precisan ser reestructurados de forma que la familia, y en especial las mujeres, puedan contar con el suficiente apoyo, ayuda, preparación y formación que proporcionen el soporte y apoyo afectivo y material necesarios.

Es igualmente importante que las personas cuidadoras dispongan de la suficiente tranquilidad para hacer frente a la situación en condiciones de ciudadanos/as libres de efectuar sus propias elecciones personales y vitales. Además, en contra de cierta perspectiva neoliberal, la intervención social en favor de las personas necesitadas de cuidados no destruye la solidaridad familiar, sino que la fortalece. Precisamente corre más riesgo de desaparecer cuando las familias se ven sobrepasadas en sus posibilidades de ofrecer el apoyo que la persona anciana necesita.

El papel que las asociaciones pueden jugar en algunos casos como el que se ha analizado en la presente investigación es una muestra de cómo la ayuda mutua, además del apoyo instrumental y afectivo que supone para las personas asociadas, puede conseguir un avance social en algunos aspectos concretos de sensibilización y mejor conocimiento social de la situación.

En el presente caso, los detalles y datos que la investigación ofrece respecto de la Asociación de Familiares de Enfermos de Alzheimer en Madrid, País Vasco y Cataluña son demostrativos de cómo la participación de la sociedad civil puede ser y es realmente efectiva y necesaria. Asimismo, se observa cómo una iniciativa que empieza a un nivel reducido e individual puede conseguir una importante repercusión social y contribuir a la mejora de algunas situaciones de especial dificultad.

Como conclusión puede decirse que las personas cuidadoras, que son principalmente mujeres, se encuentran en una situación de gran abandono por parte de los servicios sociales formales e informales, así como del sistema sanitario, que, en ocasiones y en algunos ámbitos, no proporciona la información necesaria o la ayuda esperada. Habitualmente se cuenta con el apoyo material y emocional de una red familiar reducida. La mayor parte de las familias no han solicitado siquiera ayuda, porque entienden que los servicios disponibles son escasos, y en algunos de los casos en que los han solicitado no ha sido posi- 
ble cubrir la demanda. Por otra parte, los servicios privados, cuando existen, son caros $\mathrm{y}$, en general, no se puede hacer frente al gasto económico que suponen.

El cuidado de la persona anciana enferma o dependiente en familia se asume como algo natural, como una obligación en la que el afecto y cariño están presentes. Hacer frente a los cuidados a nivel familiar da lugar a situaciones complejas en la relación entre los diferentes miembros de la red, en cuanto a la forma de hacer frente a la situación y respecto a quien hace frente de forma más directa a los cuidados que se requieren. Se han observado procesos de negociación entre los miembros de la red familiar, pareja e hijos/as, hermanos/as, yernos y nueras. También se constatan procesos de asignación cuando un miembro de la red, habitualmente una mujer, es designada por otros miembros próximos de la familia para ejercer el rol de cuidadora, a veces de forma explícita y en ocasiones de forma implícita. Se constata la construcción social del rol de cuidadora en las mujeres asumido en el proceso de socialización. Son precisamente los cambios en la estructura social, en la familia, en el mercado de trabajo, en los sistemas normativos, lo que hace pensar en que la situación actual difícilmente se podrá mantener durante mucho tiempo. Aunque parece probable que las mujeres seguirán sintiéndose más responsables y obligadas que los varones frente a las situaciones de cuidados.

Es importante señalar también una paradoja inherente a las situaciones descritas. Para conseguir la inclusión social de una parte de la población —las personas ancianas dependientes - se produce la exclusión social de otra parte de la población: las personas cuidadoras. Esas y otras situaciones resumidas en el presente artículo muestran la importancia fundamental de profundizar en algunas áreas poco conocidas, como la analizada, para poder argumentar a partir del conocimiento de la propia realidad social los verdaderos retos de la sociedad del siglo XXI. Si se desea que las familias continúen ejerciendo un intercambio de bienes y servicios entre las generaciones, se las debe proveer de los recursos necesarios. Deberán buscarse fórmulas que permitan compartir el cuidado, que incluyan como elemento irrenunciable el respeto por los derechos de todos/as los/as ciudadanos/as. Dotar a las personas de más poder de decisión y gestión es una de las vías para conseguirlo.

\section{BIBLIOGRAFIA}

Alonso, J., et al. (1990): "La salud de los ancianos en Barcelona», en Epidemiología del envejecimiento en España, Madrid: Fondo de Investigaciones Sanitarias, pp. 225-233.

Allan, Graham (1990): Family Life, Oxford: Blackwell.

Ayuntamiento de Barcelona (1994): Encuesta de Salud de Barcelona 1992-93, Barcelona: Area de Salud Pública, Ayuntamiento de Barcelona.

Bazo, María Teresa (1990): La sociedad anciana, Madrid: Centro de Investigaciones Sociológicas y Siglo XXI.

- (1991a): «Ancianidad y enfermedad», Jano: Medicina y Humanidades, 949, pp. 80-87. 
- (1991b): «La familia como elemento fundamental en la salud y bienestar de las personas ancianas", Revista Española de Geriatría y Gerontología, 1, pp. 47-52.

- (1991c): «Institucionalización de personas ancianas: un reto sociológico», Revista Española de Investigaciones Sociológicas, 53, pp. 149-164.

- (1991d): «Sociedad anciana y Estado de Bienestar», Zerbitzuan, 12-13, pp. 51-54.

- (1992a): La ancianidad del futuro, Barcelona: SG.

- (1992b): «La vejez como construcción cultural», Revista de Gerontología, 4, pp. 237-242.

- (1994): «La familia como centro privilegiado de intercambio entre generaciones», en Premios Bancaixa 1993, Valencia: Bancaixa, pp. 269-373.

- (1995): «El reto del envejecimiento: una reflexión sociológica», Revista Española de Geriatría y Gerontología, 30 (2), pp. 95-97.

Bazo, María Teresa, y Domínguez-Alcón, Carmen (1995): Cuidado informal en las personas ancianas: Familia, redes y soporte social, Madrid: Fundación Caja Madrid.

Bonvalet, C., et al. (1994): "Les moments difficiles. Léntraide au sein de la parenté selon l'enquête Proches et parents», en Actes du Seminaire International de l'AIDELF, Aranjuez.

CASTINERA, Angel (1995): «De l'Estat del benestar a la societat del benestar», Fòrum, 3, pp. 410.

Domínguez-Alcón, Carmen (1994): "Obligations familiales et solidarité», en Actes du Seminaire International de l'AIDELF, Aranjuez.

DURÁN, M.a Angeles (1992): «Salud y sociedad. Algunas propuestas de investigación», en Margarita Barañano, Mujer, trabajo y salud, Madrid: Trotta, pp. 57-79.

FINCH, Janet, y MASSON, Jennifer (1993): «Filial obligations and kind support for elderly people», en J. Bornat et al. (eds.), Community Care, Londres: MacMillan, pp. 96-106.

Gobierno Vasco (1995): Plan Gerontológico de Euskadi 1994, Vitoria: Gobierno Vasco.

INSERSO (1995a): Las personas mayores en España: Perfiles. Reciprocidad familiar, Madrid: Ministerio de Asuntos Sociales.

- (1995b): Cuidados en la vejez: El apoyo informal, Madrid: Ministerio de Asuntos Sociales.

MaYerof, Milton (1972): On Caring, Nueva York: Harper and Row.

Miguel, Jesús M. DE (1994): «Salud y sanidad», en Fundación Foessa ed., Quinto informe sociológico sobre la situación social en España: La sociedad para todos en el año 2000, Madrid: Fundación Foessa, pp. 823-1106.

MoEN, Phyllis, et al. (1992): "Successful aging: A life-course perspective on women's multiple roles and health", American Journal of Sociology, 97, 8, pp. 1612-1638.

Qureshi, Hazel, y Walker, Alan (1991): "Caring for elderly people: the family and the state», en Chris Phillipson y Alan Walker (eds.), Ageing and Social Policy: A Critical Assessment, Aldershot: Gower, pp. 109-127.

Rodríguez, Josep A. (1994): Envejecimiento y familia, Madrid: Centro de Investigaciones Sociológicas y Siglo XXI.

Roussel, Louis (1990): «Les relations intergénérationnelles au moment de la vieillesse des parents», Gerontologie et Société, 55, pp. 32-45.

SECOT (1995): Las actividades económicas de las personas mayores, Madrid: Seniors Españoles para la Cooperación Técnica.

Scitovsky, Anne A. (1994): "The High Cost of Dying Revisited», The Milbank Quarterly, 72, 4, pp. 561-591.

Ungerson, Clare (1987): Policy is Personal: Sex, Gender and Informal Care, Londres: Tavistock.

- (1993): "Caring and Citizenship: A Complex Relationship", en J. BORnAT et al., Community Care, Londres: MacMillan.

WALKER, Allan (1991): «The relationship between the family and the state in the care of older people», Canadian Journal on Aging, 10, 2. 


\title{
RESUMEN
}

En el artículo se analizan algunos resultados obtenidos en una investigación sobre cuidados familiares de salud realizada en Madrid, País Vasco y Cataluña. Son más de cien las personas analizadas y se ha utilizado la entrevista en profundidad semiestructurada. Asimismo, se han realizado tres entrevistas de grupo a personas pertenecientes a la Asociación de Familiares de Enfermos de Alzheimer en cada una de las tres comunidades. Se analizan situaciones que plantea la atención a las personas mayores, con dependencias más o menos severas, y se evidencian las repercusiones que existen a distintos niveles. Se profundiza especialmente en el impacto en las relaciones familiares, en la relación entre quien cuida y quien recibe los cuidados, en algunos factores significativos tanto en la relación entre ambos y respecto a la actividad de cuidar. Del mismo modo, se analizan ciertos aspectos referentes al impacto de esas situaciones sobre la actividad laboral y la salud de la persona cuidadora. Se concluye con algunos comentarios sobre las ayudas existentes y las perspectivas de futuro.

\begin{abstract}
Family health care and support to the elderly are explored. The research took place during the later 1994 and the beginning of the 1995 in Basque Country, Madrid and Cataluña. Qualitative methods were used based on two different samples: elderly relatives and relatives belonging to the Asociación de Familiares de Enfermos de Alzheimer (AFA). The design of the survey consisted of two parts: a) a rather long semi-structured in-depth interview addressed to 100 people caring for frail elderly relatives in the three Autonomous Communities, b) a semistructured interviews addressed to the members of AFA participating in discussion groups in the three Autonomous Communities.

Family relationships are examined: between the carer and the cared for (ambiguous feelings were found) and between female carers and their relatives (husbands, children, siblings). The lack of social services in Spain is underlined, and some current tendencies and prospects on social policy are also commented and analysed.
\end{abstract}

